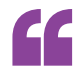

We hope that our work will convince those working in ALS that the immune system can influence disease progression

$\Rightarrow$ MOTOR NEURON DISEASE

\title{
Peripheral immune cell levels correlate with disease progression in ALS
}

Approximately $50 \%$ of patients with amyotrophic lateral sclerosis (ALS), a disease characterized by motorneuron degeneration, paralysis and ultimately death, die within 30 months of diagnosis, although some patients can survive for $>10$ years. The reasons for these variations in the rate of deterioration are largely unclear. Now, data from a longitudinal analysis of fluctuations in several peripheral immune cell populations over time demonstrate strong correlations between certain fluctuations and disease progression.

"Previous researchers have spent decades examining the role of the immune system in ALS, but only in the past 10 years or so have mouse models of ALS been used to show that changes in the immune system might have downstream effects on disease progression," explains lead author Eva Feldman.

To explore the relevance of changes in immune cell populations in patients with ALS, Feldman and colleagues obtained and analysed samples from the same patient at several time points, thus enabling longitudinal analyses to be conducted while avoiding the issues of statistical noise that impaired the conclusions of previous research.

Comparisons of immune cell subpopulations in patients with and without ALS revealed significant increases in levels of several cell subtypes, including natural killer (NK) cells and $\mathrm{CD} 16^{+}$monocytes, in the ALS group. These findings provide further confirmation of aberrant activation of the peripheral immune system in patients with ALS, although such comparisons do not reveal associations with disease progression.

When the researchers used a multivariate linear regression model to analyse changes in immune cell subtypes across samples obtained at different time points, they observed robust, statistically significant associations between both an increase in neutrophil levels and a decrease in $\mathrm{CD}^{+}{ }^{+} \mathrm{T}$ cell levels and more-rapid progression of ALS, as determined by a decrease in Revised ALS Functional Rating Scale (ALSFRS-R) score, relative to that calculated following a previous appointment. A statistically significant correlation was also observed between the accumulation of leukocytes of any subtype and disease progression.

"Many within the field believe that changes in the immune system are a consequence rather than a cause of disease progression," highlights Feldman. "We hope that our work will convince those working in ALS that the immune system can influence disease progression and can serve as a therapeutic target in the treatment of ALS."

When asked about future directions, Feldman explains that her team intend to continue to investigate the role of fluctuating peripheral immune cell populations. They plan to acquire blood samples on a monthly basis in order to better understand the short-term variations in immune cell populations that take place in patients with ALS, in addition to conducting translational investigations into the role of $\mathrm{NK}$ cells in disease progression.

Peter Sidaway

ORIGINAL ARTICLE Murdock, B. J. et al. Correlation of peripheral immunity with rapid amyotrophic lateral sclerosis progression. JAMA Neurol. http://dx.doi.org/10.1001/ jamaneurol.2017.2255 (2017) 